\title{
SCIDOC
}

\author{
International Journal of Dentistry and Oral Science (IJDOS) \\ ISSN: $2377-8075$
}

\section{Piezoelectric Internal Sinus Elevation and Simultaneous Implant Placement With Or Without Xenograft}

Research Article

Ali Silan ${ }^{1 *}$, Omar Heshmah ${ }^{1}$, Mohamad Nabough Alawa ${ }^{2}$

${ }^{1}$ Department of Oral \& Maxillofacial Surgery, Faculty of Dental Medicine, Damascus University, Damascus, Syria.

${ }^{2}$ ENT Department, Factually of Medicine, Damascus University, Syria.

\section{Abstract}

Background: Hydrodynamic piezoelectric internal sinus elevation (HPISE) is a less invasive procedure. It elevates the sinus membrane without bone compaction and postoperative vertigo caused by the mallet striking, also it reduces membrane perforation, surgical time and discomfort to the patient. Although a lot of the clinicians prefer to apply grafting materials while performing internal sinus floor elevation the radiographic analysis showed that the new bone gain in the elevated sinus was visible without grafting. This study was to evaluate the predictability of the HPISE and to compare between the two groups (with or without graft).

Purpose: The purpose of this study was to compare the results of hydrodynamic piezoelectric internal sinus elevation with and without the addition of a biomaterial xenograft.

Materials and methods: 26 dental implants in 22 patients aged 23 to 55 years participated in this randomized controlled clinical trial. After closed sinus lift operation, patients were randomly and equally divided into non-graft and xenograft graft groups. Simultaneous implant placement was then performed. Cone beam computed tomography was performed immediately and at 6 months postoperation. Bone formation was evaluated by the radiographic endo-sinus bone gain percentage around the implant.

Result: Compared with the non-graft group, the xenograft group had a significantly greater radiographic endo-sinus bone gain percentage of approximately 4 fold at 6 months postsurgery, $(\mathrm{P}<0.05)$.

Conclusion: The addition of a xenograft with piezoelectric internal sinus augmentation and simultaneous implant placement significantly enhances bone formation at 6 months postsurgery.

Keywords: Bone Formation; Bone Substitute; CBCT; Sinus Floor Elevation.

\section{Introduction}

More than half of the maxillary posterior implants were associated with a sinus augmentation procedure [1]. Sinus floor elevation either through a transcrestal approach or a lateral approach has been used for several decades to resolve vertical deficiency of atrophic posterior maxilla [2]. Although the lateral window approach has been considered to be a predictable technique for sinus augmentation, but it may cause more postoperative discomfort, such as postoperative swelling and pain, and a longer healing period than does the crestal approach [2]. So, to overcome the disadvantages of lateral window approach, variable crestal approaches, such as osteotome sinus floor elevation(OSFE) [3], bone-Added osteotome sinus floor elevation (BAOSFE) [4], Cosci Technique [5], hydraulic sinus condensing (HSC) [6], piezoelectric internal sinus elevation (PISE) [7], and hydrodynamic piezoelectric internal sinus elevation (HPISE) [8] have been introduced. In generally, the crestal approach is considered to be a less invasive procedure [2]. HPISE does not require the osteotome and mallet to break the sinus floor because it breaks it with ultrasonic vibration and elevates the sinus membrane using hydraulic pressure, without bone compaction. So we can avoid postoperative vertigo caused by the mallet striking [9], [10] in addition to that it reduces the risk of membrane perforation, surgical time and discomfort to

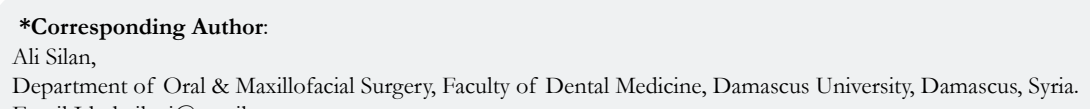

Copyright: Ali Silan ${ }^{\circ} 2021$. This is an open-access article distributed under the terms of the Creative Commons Attribution License, which permits unrestricted use, distribution and reproduction in any medium, provided the original author and source are credited. 
the patient [7]. There is still controversy regarding the necessity of adding bone substitutes in crestal sinus floor elevation [11]. Although most of the clinicians prefer to apply grafting materials while performing transcrestal sinus floor elevation [4] the original respective SFE techniques of Tatum and Summers did not use any additional grafting material in the crestal sinus floor elevation. A modified osteotome technique without grafting insertion has been described by other authors [12], [13]. Also, the radiographic analysis showed that the new bone gain in the elevated sinus was visible without grafting, ${ }^{14}$ and a histological study evidenced spontaneous new bone formation and better bone-to-implant contact for OSFE [15]. The grafting material application during BAOSFE procedure showed no advantages in histological results [16]. The aim of this study was to evaluate the predictability of the HPISE and to compare between the two groups (with or without graft).

\section{Materials and Methods}

Study Design: This study was designed as a randomized clinical study with two arms, in which patients were divided to choose the method of work randomly using the program (Research Randomizer)

The Sample: The study sample consisted of (26) dental implants in 22 patients (13 females and 7 males) whose ages ranged from 23 to 55 years, and each one of them had a unilateral or bilateral upper posterior loss and they were divided according to the method of surgical operation randomly into two groups, where the internal sinus lift operation was performed for each patient using a piezo device combined with dental implants with a xenograft graft placed in one group, while no bone graft was placed in the other group. The residual bone height RBH between the apex of the alveolar bone and the floor of the maxillary sinus ranged 5 to $7 \mathrm{~mm}$.

\section{Inclusion Criteria}

Age over 18 years and good oral health.

Absence of any of the topical contraindications (sinusitis or tumors ....).

The absence of hyperparathyroidism, or osteoporosis.

The patient should not be subjected to treatments such as corticosteroids, oral contraceptives, hormonal and chemical treatments.

They should not have a contraindication for surgery, that he had not undergone radiotherapy in the face area.

That the females are not pregnant and are not in their menstrual cycle when the surgery is performed.

That at least 6 months have passed since the last extraction in order to ensure complete healing of the bone tissue in the workplace.

After the patient was provided with an information sheet, the necessary medical approval was obtained for the surgery according to the approved medical protocol, as the surgical work was performed in the implant department and in the hospital of the Faculty of Dentistry - University of Damascus between 32018 and 92020.

\section{Radiographic Data Of The Pathological Condition:}

Before the surgery, general physical and oral examinations were performed. Dental cone beam computed tomography (CBCT) radiographs were taken using a PaX-i3D Green (Dental Pioneer, Vatech, South Korea) with fixed exposure parameters of $99 \mathrm{kV}$, $16 \mathrm{~mA}, 15 \times 15$ field of view, $0.20 \mathrm{~mm}$ slice thickness, and $9 \mathrm{sec}-$ onds images were obtained to evaluate the residual bone quantity and quality, an any possible maxillary sinus pathology.

\section{Research Materials:}

The piezo device was the Mectron (via Loreto 15/A, 16042 Carasco, Italia) with special inserts PL1, PL2, PL3, IM2P and PL2-3 for internal sinus lifting ( Fig 1), Bovine bone graft from Medpark, MBXB-P Powder with particle size 0.2 1.0mm South Korea, PT implant from Charoum, South Korea, Lidocaine $2 \%$, which contains 1: 80000 adrenaline, (New Static Colombia) and Nylon sutures from Vertmed Germany.

\section{The surgical procedure:}

All surgical procedures were performed by the same operator. After preparing the patient, sterilizing the surgical field with povidone $2 \%$ and performing local anesthesia, a surgical incision was made on the top of the socket to raise a full-thickness periosteal mucous flap while preserving the periosteum without rupture so that the bone was exposed in the target area, the implant cite was prepared using PL1 up to the sinus floor, then using PL3 for cylindrical site preparation, after that using PL2 to sinus floor consumption and membrane piezo-lift ( Fig.2 ), and then using PL3 to lift the sinus membrane by hydraulic action and bone grafting with cavitation effect. A nose-blowing test was performed after

Figure 1. Inserts of mectron piezo device.

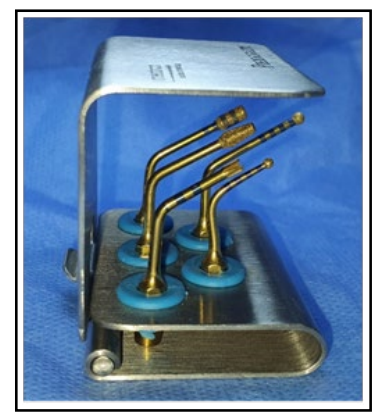


sinus elevation. After randomized sampling, a xenograft (bovine bone graft) was inserted into the sinus under the membrane in the first group, where as nothing was placed into the cavity and only a blood clot formed in the other group. A 10-mm-long implant fixture was immediately placed (Fig.3). The flap was sutured with a 4.0 Nylon suture (Vertmed, Germany). A CBCT scan was immediately performed as baseline. Postoperatively, the patient was instructed to avoid any activities causing pressure in the nasal cavity, not performing nasal blowing, opening the mouth when sneezing, not drinking with pipettes, using ice packs alternating between the two parties for a period of three hours.

$1 \mathrm{~g}$ Augmentin twice a day or $300 \mathrm{mg}$ clindamycin 3 times/day for 7 days. A nonsteroidal analgesic Flam-K (diclofenac potassium 50 mg, 3 times/day for 3 days; as needed). A $0.12 \%$ chlorhexidine mouthwash (10 mL for 1 minute, 4 times/day for 8 days starting from the second day of surgery) were provided. The patient was called to ask about any postoperative complications at day 1 and 3. Subsequently, the patient was called 7 days postoperatively for suture removal and clinical evaluation. The patients were then recalled at 6 months postoperation to assess bone formation using CBCT. During the study, the patients were instructed not to wear any denture to prevent interference with the wound healing.

\section{Radiographic Measurement and Evaluation}

The radiographic data from each patient was obtained immediately and at 6 months postoperation. The study was carried out using Ez3Di version ver.5.0.2.0 attached to the CBCT image via the sagittal and the coronal plane, considering the presence of reference points such as the implant's shoulder and the longitudinal axis passing through the center point of the implant. The alveolar bone height was measured in mesial-distal and buccal-palatal views as described by Trinh, et al [17], A1 is the distance between the mesial implant shoulder and the mesial side of the original sinus floor, and parallel to the long axis of the implant, where as $\mathrm{B} 1$ is the measurement from distal, C1 from buccal and D1 from palatal immediately postoperation. In addition, A2 is the distance between the mesial implant shoulder and the highest mesial bone margin, and parallel to the long axis of the implant, where as B2 is the measurement from distal, C2 from buccal and D2 from palatal at 6 months postoperation (Fig. 4).

The mean bone height immediately postoperation $(\mathrm{MBH} 1)=(\mathrm{A} 1$ $+\mathrm{B} 1+\mathrm{C} 1+\mathrm{D} 1) / 4$.

The mean bone height at 6 months postoperation $(\mathrm{MBH} 2=(\mathrm{A} 2$ $+\mathrm{B} 2+\mathrm{C} 2+\mathrm{D} 2) / 4$.

Endo-sinus bone gain (ESBG) was calculated as follows:

$\mathrm{ESBG}=([\mathrm{A} 2-\mathrm{A} 1]+[\mathrm{B} 2-\mathrm{B} 1]+[\mathrm{C} 2-\mathrm{C} 1]+[\mathrm{D} 2-\mathrm{D} 1]) / 4$

Radiographic data were assessed individually by two oral and maxillofacial surgeons. And the radiograph was reevaluated by the same investigators 1 weeks after the last examination. Investigators reliability measures were analyzed by Kappa statistics.

Data Analysis: Statistical analysis was performed using the IBM SPSS program for Windows (version 20, IBM, Armonk, USA). Descriptive analysis was performed. Comparisons of the mean bone height, ESBG, and \%ESBG between and with in each group

Figure 2. Use of PL2 insert.

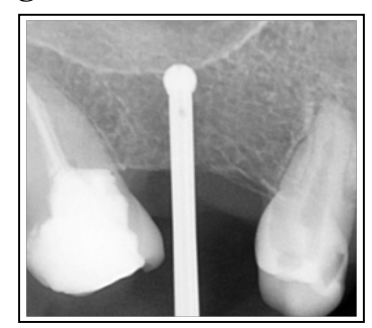

Figure 3. Implant fixture was immediately placed in xenograft group.

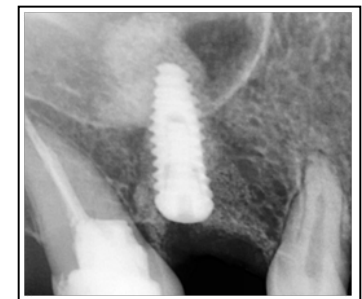

Figure 4. Measurements from mesial and distal in the two groups immediately after surgery and at 6 months postsurgery.

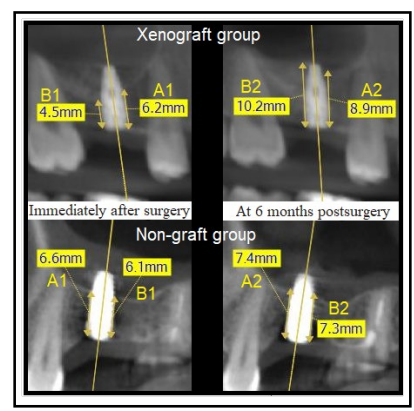


Figure 5. Descriptive analysis.

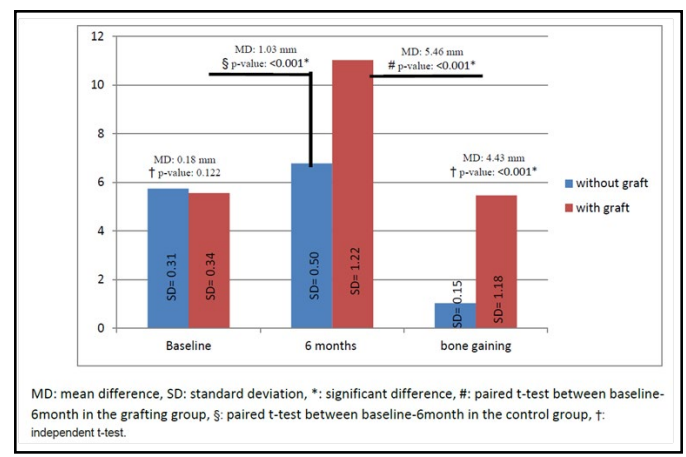

were analyzed using the independent $t$ test and Student paired t test. A value of $\mathrm{P}<0.05$ was considered statistical significant (Fig. 5).

\section{Results}

The study subjects comprised 26 implants in 22 patients $(9$ men and 13 women) with an average age 37.6 years. No patients experienced any postsurgical complications. There were 13 and 13 implants available for evaluation in the xenograft group and the non-graft group, respectively. Through the data obtained from the CBCT immediately after surgery we found that the mean bone height for the non-graft and xenograft groups was $5.74 \pm 0.31$ and $5.56 \pm 0.5 \mathrm{~mm}$, respectively. There was no significant difference in baseline mean bone height between groups ( $\mathrm{P}=0.122)$. The above is considered an indication of the homogeneity of the two groups. The non-graft and xenograft groups each had a greater mean bone height at 6-months postsurgery evaluation compared with that at baseline $(\mathrm{P}<0.05)$. So it can be said that increased alveolar bone height was radiographically observed in all subjects. New bone continuous with the sinus floor was detected around the apical end of the implant. At 6 months postsurgery, the xenograft group had an average ESBG of $5.46 \pm 1.18 \mathrm{~mm}$ while that of the control was $1.03 \pm 0.15 \mathrm{~mm}$. Moreover, the xenograft group had a significantly greater \%ESBG than that of the non-graft group of approximately 4 fold at 6 months postsurgery, $(\mathrm{P}<0.05)$.

\section{Discussion}

This study compared the results of hydrodynamic piezoelectric indirect sinus augmentation with and without the addition of a graft. Specifically, the effects of adding xenograft as a bone substitute for hydrodynamic indirect sinus augmentation. Because of the anatomical position of the maxillary bone comprised sinus wall, endo-sinus bone formation occurs when the sinus membrane is elevated. This research suggest that this process was enhanced very well when the xenograft was applied. CBCT is currently considered the imaging modality of choice for dental implant procedures and evaluation because it provides 3-dimensional measurements of the bone height in both buccal-palatal and mesial-distal views level. To increase the reliability of the measurement, 3-dimensional registration program was used [18]. Therefore, in this study, 6-month CBCT data could be accurately compared with the baseline data. The indirect technique has been recommended for sinus augmentation when the residual bone is at least 5 $\mathrm{mm}$ to stabilize the implant during the healing period [18]. The Schneiderian membrane can withstand 4 to $8 \mathrm{~mm}$ of elevation without rupture [19]. In our study, implant length of $10 \mathrm{~mm}$ was selected to minimize the risk of sinus membrane perforation and to provide space for endo-sinus bone formation. Sinus membrane perforation was not detected in both groups. Due to the innate osteogenic potential of the sinus membrane and the surrounding bony walls [20], endo-sinus bone formation was observed in both groups. The addition of a graft material significantly enhanced the amount of bone observed at 6 months. The outcome of ESBG without bone graft was limited, and the implant apex was covered by the sinus mucosa $[21,22]$. Without the addition of a bone graft material, the stability of the space underneath sinus membrane is primarily dependent on the implant apex and associated blood clot from the surgery. Early breakdown of the blood clot during healing process can result in membrane collapse and limit the amount of bone formation. The addition of a bone substitute provides an osteoconductive scaffold and space maintenance to support the Schneiderian membrane and prevent premature collapse. Adding bone graft resulted in the higher mean bone gain percentage than without graft [23]. The authors stated that this could be attributed to use of graft. In our study, the mean bone gain of the indirect sinus lift without a bone graft was $1.04 \pm 0.14$ $\mathrm{mm}$ at the 6-month evaluation, It is less than similar studies in which the maxillary sinus lift was performed by osteotome, where one study found $2.4+0.4 \mathrm{~mm}$ after 6 months of surgery [17], as well as a study in which there was an increase in bone of $1.8 \mathrm{~mm}$ in two years of observation [24]. This can be attributed to the fact that in the osteotome technique, $2 \mathrm{~mm}$ of maxillary sinus floor is broken and pushed into the pocket, and this can be considered as an autologous bone graft that can increase the proportion of bone morphology inside the sinus, while in the piezo technique, the sinus floor is removed and no part of it is entered with in the sinus. Even though the endo-sinus bone can be gained without adding a bone graft, the bone gain is very limited. In contrast, we found that the xenograft group induced more endo-sinus bone formation around the implant than that of the non-graft group at 6 months postoperation. Our clinical result supports the use of a graft material in the piezoelectric indirect sinus augmentation. Long-term follow-up to determine the survival rate of the sinus augmentation and implant should be performed in future studies.

\section{References}

[1]. Seong WJ, Barczak M, Jung J, Basu S, Olin PS, Conrad HJ. Prevalence of sinus augmentation associated with maxillary posterior implants. J Oral Implantol. 2013 Dec;39(6):680-8. Pubmed PMID: 21651386.

[2]. Kim JM, Sohn DS, Bae MS, Moon JW, Lee JH, Park IS. Flapless transcrestal sinus augmentation using hydrodynamic piezoelectric internal sinus elevation with autologous concentrated growth factors alone. Implant Dent. 2014 Apr;23(2):168-74. Pubmed PMID: 24637529.

[3]. Summers RB. A new concept in maxillary implant surgery: the osteotome 
technique. Compendium. 1994 Feb;15(2):152, 154-6, 158 passim; quiz 162.Pubmed PMID: 8055503.

[4]. Summers RB. The osteotome technique: Part 2--The ridge expansion osteotomy (REO) procedure. Compendium. 1994 Apr;15(4):422, 424, 426, passim; quiz 436. Pubmed PMID: 8055514.

[5]. Cosci F, Luccioli M. A new sinus lift technique in conjunction with placement of 265 implants: a 6-year retrospective study. Implant Dent. 2000;9(4):363-8. Pubmed PMID: 11307560.

[6]. Chen L, Cha J. An 8-year retrospective study: 1,100 patients receiving 1,557 implants using the minimally invasive hydraulic sinus condensing technique. J Periodontol. 2005 Mar;76(3):482-91. Pubmed PMID: 15857085.

[7]. Sohn DS, Lee JS, An KM, Choi BJ. Piezoelectric internal sinus elevation (PISE) technique: a new method for internal sinus elevation. Implant Dent. 2009 Dec;18(6):458-63. Pubmed PMID: 20009598.

[8]. Kim JM, Sohn DS, Heo JU, Park JS, Jung HS, Moon JW, et al. Minimally invasive sinus augmentation using ultrasonic piezoelectric vibration and hydraulic pressure: a multicenter retrospective study. Implant Dent. 2012 Dec;21(6):536-42. Pubmed PMID: 23149505.

[9]. Kim MS, Lee JK, Chang BS, Um HS. Benign paroxysmal positional vertigo as a complication of sinus floor elevation. J Periodontal Implant Sci. 2010 Apr;40(2):86-9. Pubmed PMID: 20498765.

[10]. Peñarrocha M, Garcia A. Benign paroxysmal positional vertigo as a complication of interventions with osteotome and mallet. J Oral Maxillofac Surg. 2006 Aug;64(8):1324; author reply 1324. Pubmed PMID: 16860237.

[11]. Younes R, Nader N, Khoury G, editors. Sinus Grafting Techniques: A Stepby-Step Guide. Springer; 2015 Jan 21.

[12]. Pjetursson BE, Rast C, Brägger U, Schmidlin K, Zwahlen M, Lang NP. Maxillary sinus floor elevation using the (transalveolar) osteotome technique with or without grafting material. Part I: Implant survival and patients' perception. Clin Oral Implants Res. 2009 Jul;20(7):667-76. Pubmed PMID: 19486079.

[13]. Schmidlin PR, Müller J, Bindl A, Imfeld H. Sinus floor elevation using an osteotome technique without grafting materials or membranes. Int J Periodontics Restorative Dent. 2008 Aug;28(4):401-9. Pubmed PMID: 18717379.

[14]. Lai HC, Zhuang LF, Lv XF, Zhang ZY, Zhang YX, Zhang ZY. Osteotome sinus floor elevation with or without grafting: a preliminary clinical trial. Clin Oral Implants Res. 2010 May;21(5):520-6. Pubmed PMID: 20337666.

[15]. Rawat A, Thukral H, Jose A. Indirect Sinus Floor Elevation Technique with Simultaneous Implant Placement without Using Bone Grafts. Ann Maxil- lofac Surg. 2019 Jan-Jun;9(1):96-102. Pubmed PMID: 31293935.

[16]. S Si MS, Zhuang LF, Gu YX, Mo JJ, Qiao SC, Lai HC. Osteotome sinus floor elevation with or without grafting: a 3-year randomized controlled clinical trial. J Clin Periodontol. 2013 Apr;40(4):396-403. Pubmed PMID: 23425152.

[17]. Trinh HA, Dam VV, Le B, Pittayapat P, Thunyakitpisal P. Indirect Sinus Augmentation With and Without the Addition of a Biomaterial: A Randomized Controlled Clinical Trial. Implant Dent. 2019 Dec;28(6):571-577. Pubmed PMID: 31567794.

[18]. Agamy EM, Niedermeier W. Indirect sinus floor elevation for osseointegrated prostheses. A 10-year prospective study. J Oral Implantol. 2010;36(2):11321. Pubmed PMID: 20426588

[19]. Reiser GM, Rabinovitz Z, Bruno J, Damoulis PD, Griffin TJ. Evaluation of maxillary sinus membrane response following elevation with the crestal osteotome technique in human cadavers. Int J Oral Maxillofac Implants. 2001 Nov-Dec;16(6):833-40. Pubmed PMID: 11769834.

[20]. Simion M, Fontana F, Rasperini G, Maiorana C. Long-term evaluation of osseointegrated implants placed in sites augmented with sinus floor elevation associated with vertical ridge augmentation: a retrospective study of 38 consecutive implants with 1- to 7-year follow-up. Int J Periodontics Restorative Dent. 2004 Jun;24(3):208-21. Pubmed PMID: 15227769.

[21]. Sul SH, Choi BH, Li J, Jeong SM, Xuan F. Histologic changes in the maxillary sinus membrane after sinus membrane elevation and the simultaneous insertion of dental implants without the use of grafting materials. Oral Surg Oral Med Oral Pathol Oral Radiol Endod. 2008 Apr;105(4):e1-5. Pubmed PMID: 18329561.

[22]. Sul SH, Choi BH, Li J, Jeong SM, Xuan F. Effects of sinus membrane elevation on bone formation around implants placed in the maxillary sinus cavity: an experimental study. Oral Surg Oral Med Oral Pathol Oral Radiol Endod. 2008 Jun;105(6):684-7. Pubmed PMID: 18299220.

[23]. Nedir R, Nurdin N, Khoury P, Bischof M. Short Implants Placed with or without Grafting in Atrophic Sinuses: The 3-Year Results of a Prospective Randomized Controlled Study. Clin Implant Dent Relat Res. 2016 Feb;18(1):10-8. Pubmed PMID: 25622803.

[24]. Brizuela A, Martín N, Fernández-Gonzalez FJ, Larrazábal C, Anta A. Osteotome sinus floor elevation without grafting material: Results of a 2-year prospective study. J Clin Exp Dent. 2014 Dec 1;6(5):e479-84. Pubmed PMID: 25674312. 